

\title{
The Fermilab g-2 Experiment
}

\section{Themis Bowcock}

University of Liverpool

Oliver Lodge Laboratory, Oxford St., Liverpool, L69 7ZE, UK

E-mail: bowcockeliverpool.ac.uk

The g-2 experiment at Fermilab is due to start taking data in 2017. The experiment will make new measurements of the muon anomalous moment, $a_{\mu}$, and its electric dipole moment, $d_{\mu}$. Compared to its predecessor, E821 at Brookhaven National Laboratory, the new experiment aims to improve its precision on $a_{\mu}$ approximately fourfold and the sensitivity to $d_{\mu}$ by over an order of magnitude. The g-2 experiment will provide vital information on the existing discrepancy between measurement and the Standard Model prediction of the anomalous magnetic moment. We summarize the status of preparation of the experiment, that of the underlying theory and the $e^{+} e^{-}$measurements required to interpret the result.

Flavor Physics and CP Violation

6-9 June 2016

Caltech, Pasadena CA, USA

\footnotetext{
${ }^{1}$ Speaker

(C) Copyright owned by the author(s) under the terms of the Creative Commons

Attribution-NonCommercial-NoDerivatives 4.0 International License (CC BY-NC-ND 4.0).
} 


\section{Introduction}

Polarized muon beams have been used to make sub-percent measurements of the muon magnetic moment for approximately 60 years. The first sub-percent measurement was performed in $1957^{[1]}$. By 1965 CERN had performed the first in a series of three experiments. Using the Synchro-Cyclotron ${ }^{[2]}$ they achieved $0.1 \%$ precision on the the muon anomalous moment, $a_{\mu}$. This demonstrated that the famous Schwinger ${ }^{[3]}$ correction $\mathrm{O}(\alpha)$ to $a_{\mu}$. It is interesting to note that the same team also set a limit on the electric dipole moment, $d_{\mu}$ of $\sim<10^{-17} \mathrm{e} \mathrm{cm}$. The second experiment, the first using a muon storage ring ${ }^{[4]}$, achieved a precision of approximately $250 \mathrm{ppm}$ for $a_{\mu}$, for both $\mu^{\ddagger}$. This, at the time, agreed with theoretical prediction, if the light-by-light scattering process were correctly taken into account ${ }^{[5]}$ as well as vacuum polarization and $4^{\text {th }}$ order QED processes. Driven by the need for greater precision ${ }^{[6]}$, the quest to understand the muon-electron mass difference and the wish to test the limits of QED, a third CERN experiment was proposed. This experiment was the prototype of the Brookhaven ${ }^{[7,8]}$ E821 experiment, that used the magic momentum technique (see section 2.1) to dramatically reduced systematic uncertainties. The E821 experiment achieved ${ }^{[9,10]}$ an uncertainty of $540 \mathrm{ppb}$ on $a_{\mu}$; this is arguably the most precise measurement in High Energy Physics. Currently experimental measurement and standard model (SM) predictions differ by $\sim 3.4 \sigma$. Given the potential importance of a divergent SM and experimental value, a new $\mathrm{g}-2$ experiment has been proposed ${ }^{[11]}$ and is under construction at FNAL. The new experiment aims to increase the experimental precision by a factor of four. Together with theoretical expected improvements, which reduce the $\mathrm{SM}^{[12]}$ uncertainty $\delta a_{\mu}^{S M}$ from $\mathrm{O}(50)$ to $\mathrm{O}(35) \times 10^{-11}$, confirmation of the central value of $a_{\mu}$ obtained by E821 would have a significance of over $7 \sigma-$ this makes g-2 one of the most important tests of the SM currently in preparation. The existing world limit on $d_{\mu}$ of $\left|d_{\mu}\right|<1.8 \times 10^{-19} \mathrm{e} \mathrm{cm}$ will also be improved, by over an order of magnitude, by the new FNAL experiment.

\section{The g-2 experiment at Fermilab}

The experiment builds on the expertise E821 experiment and aims to reduce both statistical and systematic errors; it will collect 20 times the statistics of the Brookhaven experiment and is designed to reduce the errors to the $100 \mathrm{ppb}$ level.

\subsection{Measurement Technique}

The experimental method is well described in the FNAL g-2 Technical Design Report $^{[11]}$. As with previous experiments polarized muons are injected into a storage ring (see section 2.2). As the muons travel round the ring the muon polarizations rotaterelative to the momentum at a rate, $\omega_{a}$. This frequency is related to the spin and cyclotron frequencies through $\omega_{a}=\omega_{S}-\omega_{C}=-a \frac{Q e}{m} B$, where we note that $\omega_{a}$ depends on the magnetic field in the ring. An electric field is required to focus the muons and, under these conditions, the expression $\omega_{a}$ depends on the momentum, $p$, and the electric field:

$$
\vec{\omega}_{a}=-\frac{Q e}{m}\left[a_{\mu} \vec{B}-\left(a_{\mu}-\left(\frac{m c}{p}\right)^{2}\right) \frac{\vec{\beta} \times \vec{E}}{c}\right]
$$


This expression remains simple when the momentum of the muons used is the "magic momentum" $p_{\text {magic }}=\frac{m}{\sqrt{a_{\mu}}} \cong 3.09 \mathrm{GeV} / \mathrm{c}$, corresponding to a laboratory lifetime of $64 \mu$ s. Under these conditions, to first order, the effects of the electric fields can be ignored.

The magnetic field in the experiment will be measured using proton nuclear magnetic resonance. The Larmor frequency of the proton is related to the magnetic field by $\hbar \omega_{p}=2 \mu_{p}|\vec{B}|$. The anomalous magnetic moment is extracted using

$$
a_{\mu}=\frac{\omega_{a} / \omega_{p}}{\lambda_{+}-\omega_{a} / \omega_{p}}
$$

Where $\lambda_{+}=\mu_{\mu^{+}} / \mu_{p}$ is the ratio of muon and proton magnetic moments and is determined from muonium ${ }^{[13]}$ to $27 \mathrm{ppb}$.



Figure 1: cosine of the angle between the positron from muonspin as a function of energy. The highest energy positrons are strongly correlated with themuon spin.



Figure 2: Modulation of the hits in the E821 calorimeters as a function of time.

The (positive) muon decays through the process $\mu^{+} \rightarrow e^{+}+\bar{v}_{\mu}+v_{e}$. The highest energy positrons are highly correlated with the muon spin(see Figure 1). Thus if we detect the decay positrons, as the muon spin rotates, their number emitted in the forward direction will be modulated by $\omega_{a}$. By placing calorimeters around the ring (see section Error! Reference source not found.) the experiment will observe rate of positrons which is a convolution of the muon exponential decay and an amplitude beating with frequency $\omega_{a}$. The rate can be approximately parameterized by the form:

$$
N(t)=N_{0} e^{\left(-t / \gamma \tau_{\mu}\right)}\left[1-A \cos \left(\omega_{a} t+\phi\right)\right] .
$$

This approximately describes the rate observed at E821(see Figure 2).

\subsection{Muon Delivery and the Storage Ring}

The increase in statistics required by the g- 2 experiment will be made by the relocation of the E821 to the new FNAL Muon Cmpus and provisioned by a high intensity proton beam from the Booster $\left(\sim 10^{13}\right.$ protons on target /s). Pions with a momentum close to 3.1 $\mathrm{GeV}$ are directed out of the target hall through a beamline with a high muon capture 
efficiency and injected in the Delivery Ring (see Figure 3). The muons sample is



Figure 3: Schematic of FNAL muon delivery system



Figure 4: Schematic of the Storage Ring. Muons are injected at the "top" of ring. Shown are the positions of the electrostatic quadrupoles Q1-Q4, and the kickers (K1-K3) which correct the orbit.

purified by circulating the beam in the Delivery Ring and separating protons from muons, via time of flight, prior to a kicker safely ejecting the protons. The muons are brought, via a new beamline, to the Storage Ring (see Figure 4) which resides in a custom building that controls temperature and a stable platform for the ring.

The muons are injected into the ring, via the inflector magnet. Kicker magnets are then used to place the muons in the required orbit. Vertical focussing of the beam is provided via electrostatic quadrupoles ${ }^{[14]}$ and they are kept in a circular orbit by a $1.45 \mathrm{~T}$ vertical magnetic field. The magnet (Figure 5) is designed to provide an extremely even field within a $9 \mathrm{~cm}$ circular envelope that contains the muon "torus". The field is provided by a super-conducting magnet designed ${ }^{[15-17]}$ to permit passive shimming of the pole pieces with additional steel wedges and foils. Active shims (printed circuit boards and coils) are provided. The field uniformity is expected to be $\mathrm{O}(1 \mathrm{ppm})$.



Figure 5: Diagram showing cross -section of the Storage Ring (from Ref. [11]). Absolute knowledge of the field within the ring as a function of position is expected to be better than about $35 \mathrm{ppb}$. Overall improvements to the field related systematics on $\omega_{p}$ are expected to be over a factor of two from E821 from 170 ppb to about 70 ppb.

\subsection{Calorimeters and Trackers}

New detectors are being installed in the g-2 experiment to improve the reconstruction of the positrons. Most important are new calorimeters ${ }^{[18-21]}$. The requirements on the calorimeters are:

- Energy resolution of better than $5 \%$ at $2 \mathrm{GeV}$

- Timing resolutions of better than $100 \mathrm{ps}$ 
- $100 \%$ resolution of two showers with time separations of $5 \mathrm{~ns}$

- Gain stability of $<0.1 \%$ over $200 \mu$ s and long term stability $<1 \%$.

To provide this 24 segmented $\mathrm{PbF}_{2}$ calorimeters are being built (see Figure 6). These consist of $6 \times 9$ arrays of crystals which have dimension $25 \times 25 \times 140 \mathrm{~mm}^{3}$. These will be evenly spaced round the Storage Ring. Each $\mathrm{PbF}_{2}$ crystal will be readout by a 16channel Hamamatsu Photonics MPPC. To provide, and monitor, stability a laser calibration system will also be installed.



Figure 6:Photograph of a g-2 caorimeter.



Figure 7: Photograph of tracker module. There are 8 in a complet tracker.

In front of three of the calorimeters trackers stations will be installed a tracker. Each tracker is composed of 8 modules. The modules are straw detectors with a double U-V layers with the stereo-angle being $15^{\circ}$. Each module contains $128 \sim 10 \mathrm{~cm}$ long, $5 \mathrm{~mm}$ diameter straws. The straws are indentical those planned for the mu2e experiment. The readout is via a 25 micron gold tungsten wire strung at approximately $30 \mathrm{~g}$ of tension. Resolutions of 100 micron per layer were achieved in test beam. The tracker stations will measure the beam profile throughout the fill. They will give information on:

- Off magic-momentum muons and non-perpendicar motion of the muons

- Betatron motion that changes acceptance of the calorimetrers

- The muon spatial distribution and information on lost muons.

They will also provide infrmation useful to understand calorimeter pileup and gain. The tracking detectors are unique in that they can provide an independent technique to measure the muon $\mathrm{EDM}^{[22]}$.

\subsection{Expected sources of systematic and statistical uncty}

The new detectors serve to address key sources of systematic error and contribute to reducing the new g- 2 experimental uncertaities to $\mathrm{O}(70) \mathrm{ppb}$.

\begin{tabular}{l|l|l|l}
\hline E821 & Size $(\mathrm{ppm})$ & Plan FNAL g-2 & Goal $(\mathrm{ppm})$ \\
\hline Gain Changes & 0.12 & Better calo. & 0.02 \\
Lost muons & 0.09 & Trackers & 0.02 \\
Pileup & 0.08 & Better Calo \& Trackers & 0.04 \\
Coherent Betatron & 0.07 & Trackers & 0.03 \\
Oscillations & & & \\
E-field/Pitch & 0.06 & Trackers & 0.03 \\
Diff. Decay & 0.05 & Better & 0.02 \\
& & Kicker/Simulation & \\
\hline Total & 0.20 & & 0.07 \\
\hline
\end{tabular}

Table 1: Systematic uncertainties at E821 and expected impact on $\omega_{\mathrm{a}}$. Extracted from Ref. [11]. 


\section{Theoretical Developments}

The deviation of the measured $a_{\mu}$ from SM expectation remains a source of experimental interest. The SM expectation is depends on a number of large corrections:

- The QED uncertainty known to a high level of precision and which has been calculated to $10^{\text {th }}$ order. $^{[23-27]}$

- ElectroWeak corrections. ${ }^{[28-31]}$

- Hadronic uncertainties due to photons coupling to the vacuum. ${ }^{[32-40]}$

These corrections and their associated uncertainties are shown graphically in Error! Reference source not found. and enumerated in Table 2. Current best estimates give

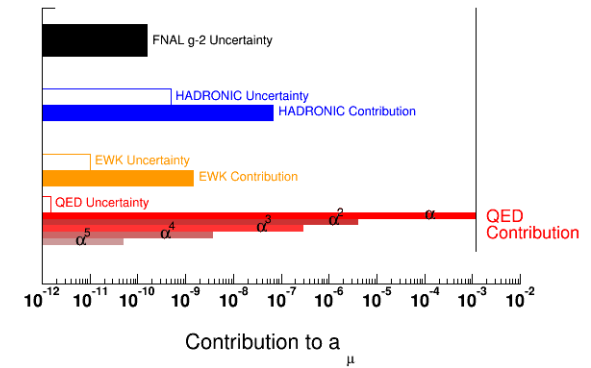

Figure 8: Gaphical representation of corrections to $a_{\mu}$ and their uncertainties.

\begin{tabular}{l|c|c}
\hline & $\begin{array}{c}\text { Now } \\
\left(\times 10^{-11}\right)\end{array}$ & 2018 \\
\hline QED & 0.08 & 0.08 \\
\hline EW & 1.0 & 1.0 \\
\hline HVP - lo & 43 & 26 \\
\hline HVP - ho & 0.7 & 0.7 \\
\hline HLBL & 26 & 26 \\
\hline Total & 50 & 36 \\
\hline
\end{tabular}

Table 2: Current theoretical uncertainties on $a_{\mu}$ and the expected improvements

$\Delta \alpha_{\mu}=(286 \pm 80) \times 10^{-11}$.

The corrections that dominate are the hadronic vacuum polarization. These are due to processes like that drawn in Figure 9. These are calculated using the classic dispersion relation formula:

$$
a_{\mu}^{H L O}=\frac{1}{4 \pi^{3}} \int_{4 m_{\pi}^{2}}^{\infty} d s K(s) \sigma(s) .
$$

In order to account for the anomalous magnetic moment the measured cross-sections would have to be heavily underestimated. Approximately $75 \%$ of the contribution is from the $\pi^{+} \pi^{-}$cross section and over $90 \%$ of that is below $1.8 \mathrm{GeV}$. To correct the value of the anomalous magnetic moment the $e^{+} e^{-}$crosssection would have to be changed (upwards) by over $6 \%$ in the region of the $\omega$ and $\rho$ resonances. Existing data does not support this level of systematic uncertainty, and new data from $\mathrm{KLOE}^{[41]}$ shows no evidence of anomalies. Furthermore if the hadronic

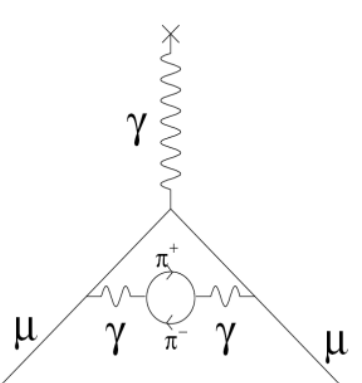

Figure 9: Typical HVP correction term cross-section date were "wrong" this would substantially increase tension in the EW precision fits ${ }^{[42]}$, and specifically with the observed mass of the Higgs. There are suggestions that new independent interpretations ${ }^{2}$

\footnotetext{
${ }^{2}$ Private communications, T. Teubner
} 
of $e^{+} e^{-} \rightarrow \mu^{+} \mu^{-}$could be used to shed further light on this problem. But these have not yet been published.The current consensus is there no immediate movement in the value of the hadronic vacuum polariyation theoertical corrections.

\subsection{Lattice Gauge Calculations}

Substantial work has been performed over the last few years on the lattice that impacts directly (and independently) on the prediction of $a_{\mu}{ }^{[43-46]}$. Recent papers ${ }^{[47,48]}$ suggest the lattice average inceases the discrepancy between the SM and the E821 results; using the lattice the significance stands at almost $4 \sigma$.

\subsection{Interpretion of Results}

The theoretical and experimental work over the last decade has tended to make the E821 result more significant. There have been several attempts to explain the anomaly in terms of NP. These vary from extension of the $\mathrm{SM}^{[49,50]}$, to axions ${ }^{[51]}$, to dark particles $^{[52-54]}$. Without the FNAL g-2 experimental measurements, and with the lack of evidence of NP physics from the LHC, it remains fascinating but premature to speculate on the cause of the anomaly. The tension of the E821 results with the SM stands as one of the most important in current physics.

\section{Summary}

We have provided a brief description of the g-2 experiment at FNAL due to take first data in 2017. It builds on the seminal E821 experiment at Brookhaven and aims to resolve the outstanding discrepancy between the measurement of the anomous magnetic moment of the muon and the SM. The experiment will also provide the best measurements to date of the muon electric dipole moment.

\section{References}

[1] J. M. Cassels, et al., Experiments with a Polarized Muon Beam Proc. Phys. Soc. A 70(1957)543.

[2] G. Charpak, et al., The anomalous magnetic moment of the muon, Nuovo Cim. 37(1965)1241.

[3] J. S. Schwinger, On Quantum-Electrodynamics and the Magnetic Moment of the Electron, Phys. Rev. 73(1948) 416.

[4] J. Bailey, et al., Precise Measurement of the Anomalous Magnetic Moment of the Muon, Nuovo Cim. A9(1972)369.

[5] J. Aldins, et al., Photon-Photon Scattering Contribution to Sixth-Order Magnetic Moments of Muon and Electron, Phys. Rev. D 1(1970)2378.

[6] F. Combley, et al., The CERN Muon (g-2) Experiments Physics Reports 68(1981)93.

[7] V. W. Hughes, et al., A Possible Higher Precision Measurement of the muon g-2 value, AIP Conf.Proc. 123(1984)

[8] C. S. Ozben, et al., Muon g-2 experiment at Brookhaven National Laboratory, Nucl.Phys.Proc.Suppl. 105(2002)

[9] G. W. Bennett, et al., Measurement of the negative muon anomalous magnetic moment to 0.7 ppm, Phys.Rev.Lett. 92(2004)

[10] G. W. Bennett, et al., Final Report of the Muon E821 Anomalous Magnetic Moment Measurement at BNL, Phys.Rev. D73(2006)

[11] J. Grange, et al., Muon (g-2) Technical Design Report, (2015)

[12] T. Teubner, et al., g-2 of the muon and Delta alpha re-evaluated, Nucl.Phys.Proc.Suppl. 218(2011)

[13] W. Liu, et al., High precision measurements of the ground state hyperfine structure interval of muonium and of the muon magnetic moment, Phys. Rev. Lett. 82(1999)711.

[14] G. Bennett, et al., Electrostatic quadrupole focusing in the AGS g-2 storage ring, Conf.Proc. C930517(1993) 
[15] G. T. Danby, et al., Shimming techniques for the ultraprecise muon g-2 storage ring at the AGS, IEEE Trans.Magnetics 30(1994)

[16] G. Danby, et al., The Brookhaven muon storage ring magnet Nucl.Instrum.Meth. 457(2001)151.

[17] D. Brown, et al., Ultraprecise superferric storage ring magnet for the muon g-2 experiment, IEEE Trans.Magnetics 24(1988)

[18] R. McNabb, Muon g-2 Calorimeter Prototype, (2007)

[19] C. Polly, et al., Muon g-2 Calorimeter Prototypes, (2010)

[20] A. Chapelain, Development of the electromagnetic calorimeter waveform digitizers for the Fermilab Muon g-2 experiment, PoS EPS-HEP2015(2015)

[21] A. Anastasi, et al., Test of candidate light distributors for the muon ( $g-2)$ laser calibration system, Nucl.Instrum.Meth. A788(2015)

[22] G. W. Bennett, et al., An Improved Limit on the Muon Electric Dipole Moment, Phys.Rev. D80(2009)

[23] T. Kinoshita, Radiative corrections of eighth-and tenth-orders to lepton g-2, Nucl.Phys.Proc.Suppl. 157(2006)

[24] T. Aoyama, et al., Complete Tenth-Order QED Contribution to the Muon g-2, Phys.Rev.Lett. 109(2012)

[25] M. Hayakawa, et al., Tenth-Order QED Contribution to the Lepton Anomalous Magnetic Moment -- Sixth-Order Vertices Containing an Internal Light-by-Light-Scattering Subdiagram, Phys.Rev. D85(2012)

[26] T. Aoyama, et al., Tenth-Order QED Lepton Anomalous Magnetic Moment --- Eighth-Order Vertices Containing a Second-Order Vacuum Polarization, Phys.Rev. D85(2012)

[27] T. Kinoshita, Tenth-order QED contribution to the electron g-2 and high precision test of quantum electrodynamicS, Int.J.Mod.Phys. A29(2014)


Phys.Lett. B649(2007)

[29] T. Teubner, et al., Update of g-2 of the Muon and Delta Alpha, Chin.Phys. C34(2010)

[30] K. Hagiwara, et al., (g-2)_mu and alpha(M_Z^2) re-evaluated using new precise data, J.Phys. G38(2011)

[31] T. Teubner, et al., g-2 and alpha(M**2(Z)): Status of the standard model predictions, Nucl.Phys.Proc.Suppl. 225-227(2012)

[32] S. Bodenstein, et al., Hadronic contribution to the muon g-2 factor, Phys.Rev. D88(2013)

[33] A. Nyffeler, Hadronic light-by-light scattering in the muon g-2: impact of proposed measurements of the $\pi^{0} \rightarrow \gamma \gamma$ decay width and the $\gamma^{*} \gamma \rightarrow \pi^{0}$ transition form factor with the KLOE2 experiment, PoS CD12(2013)

[34] M. Davier, New Results on the Hadronic Vacuum Polarization to the Muon g-2, Chin.Phys. C34(2010)

[35] M. Davier, et al., Reevaluation of the hadronic contribution to the muon magnetic anomaly using new $e^{+} e^{-} \rightarrow \pi^{+} \pi$ cross section data from BABAR, Eur.Phys.J. C66(2010)

[36] D. Moricciani, Hadronic light-by-light contribution to the muon anomalous magnetic moment, J.Phys.Conf.Ser. 349(2012)

[37] S. Eidelman, Muon g-2 and Hadronic Vacuum Polarization: Recent Developments, EPJ Web Conf. 118(2016)

[38] G. Venanzoni, Measuring the Leading Order Hadronic Contribution to the Muon g-2 in the Space-like Region, Frascati Phys.Ser. 61(2016)

[39] G. Colangelo, et al., Dispersion relation for hadronic light-by-light scattering and the muon g-2, PoS CD15(2016)

[40] F. Jegerlehner, Leading-order hadronic contribution to the electron and muon $\$ g-2 \$, E P J$ Web Conf. 118(2016)

[41] P. Moskal, et al., Recent results on hadron physics at KLOE, (2013)

[42] M. Passera, et al., The Muon g-2 and the bounds on the Higgs boson mass, Phys.Rev. D78(2008)

[43] M. Gockeler, et al., Vacuum polarization and the muon g-2 in lattice QCD, Nucl.Phys.Proc.Suppl. 129(2004)

[44] X. Feng, et al., Hadronic Vacuum Polarization Contribution to $g$-2 from the Lattice, Nucl.Phys.Proc.Suppl. 225-227(2012)

[45] E. Shintani, Progress of lattice calculation of light-by-light contribution to muon $g-2$, Nucl.Part.Phys.Proc. 273-275(2016)

[46] M. Benayoun, et al., A BHLS model based moment analysis of muon g-2, and its use for lattice 
QCD evaluations of $a_{\mu}$ (2016)

[47] A. Chakraborty, et al., Probing g-2 at the LHC in the paradigm of $\$ R \$$-parity violating MSSM, Phys.Rev. D93(2016)

[48] B. Chakraborty, et al., The hadronic vacuum polarization contribution to \$a_\mu $\$$ from full lattice QCD, (2016)

[49] A. Kobakhidze, et al., Probing the MSSM explanation of the muon g-2 anomaly in dark matter experiments and at a $100 \mathrm{TeV}$ pp collider, (2016)

[50] N. Okada, et al., 125 GeV Higgs boson mass and muon g-2 in 5D MSSM, (2016)

[51] W. J. Marciano, et al., Light-by-light contributions of axion-like particles to lepton dipole moments, (2016)

[52] K. Harigaya, et al., Light Chiral Dark Sector, Phys.Rev. D94(2016)

[53] J. Kile, et al., Lepton-Flavored Dark Matter, Phys.Lett. B744(2015)

[54] C.-Y. Chen, et al., Implications of a light "dark Higgs" solution to the g-2 discrepancy, Phys.Rev. D93(2016) 